\title{
Der Streit zwischen der theologischen und der philosophischen Fakultät in Kants Schrift "Der Streit der Fakultäten"
}

DOI: http://dx.doi.org/10.12775/RF.2016.036

\section{Politischer Religionskonflikt und Zensurprivileg}

Kants Spätwerk „Der Streit der Fakultäten in drei Abschnitten“ (1798) ${ }^{1}$ ist aus der Perspektive einer Theorie der Philosophie in der institutionellen Verankerung eines Universitätsmodells in Bezug auf die preußische Kulturpolitik des ausgehenden 18. Jahrhunderts verfaßt. Dennoch beinhaltet diese Schrift keine Meta-Reflexion in Form eines unmittelbaren Reflexes auf die viergliedrige Fakultätenstruktur, die ein historisches Charakteristikum der preußischen Universitäten war. Wir haben es vielmehr mit einer philosophischen Theorie zu tun, die um das Selbstverständnis des Gegenstandes ihrer eigenen Wissenschaft ringt, und zwar: einerseits in Abgrenzung von und in Anknüpfung an die Gegenstände der benachbarten Disziplinen innerhalb derselben Universitätshierar-

1 Über Abweichungen gegenüber der ersten Fassung von 1794 sowie zu Vorarbeiten, Entwicklung des Manuskriptes und publikationstechnischen Fragen vgl. R. Brandt: Zum „Streit der Fakultäten“. In: R. Brandt / W. Stark (Hrsg.), Neue Autographen und Dokumente zu Kants Leben, Schriften und Vorlesungen. Hamburg 1987 (Kant-Forschungen Bd. 1), 31-78. - Kants Werke werden im folgenden zitiert nach den von der königlich preußischen bzw. Berlin-Brandenburgischen Akademie der Wissenschaften hrsg. Gesammtelten Schriften, unter Angabe der Band-, Seiten- und Zeilenzahl. Die Kritik der Urteilskraft (KU) zitiere ich nach der Ausgabe von Heiner F. Klemme, Hamburg 2006. 
chie (Theologie, Jurisdiktion, Medizin), andererseits und zugleich - stellvertretend für alle Fakultäten - in der Verteidigung der Autonomie und der Rechte der Universität insgesamt gegen staatliche Eingriffe in die Selbstverwaltung und in der Gehorsamspflicht zur bestmöglichen, loyalitätskonformen Ausbildung von Staatsbeamten.

In Anbetracht der engen Grenzen, die einem Konferenzvortrag vorbehalten sind, möchte ich mich ausschließlich auf den Streit der philosophischen mit der theologischen Fakultät beschränken. Dieser Streit wird unmittelbar tangiert von Kants eigenem Konflikt mit der staatlichen Obrigkeit im Zusammenhang mit der Zensur seiner Religionsschrift. Bereits die Vorrede zur Fakultätenschrift dokumentiert diesen Zusammenhang und macht damit die Bedeutung des politischen Religionskonflikts sichtbar $^{2}$. Es ist deshalb zum näheren Verständnis des ersten Abschnitts des "Streits der Fakultäten“ ratsam, Kants Schrift über die Religion innerhalb der Grenzen der bloßen Vernunft (1793) in die Betrachtung mit einzubeziehen, zumal Kant zeitweilig eine kombinierte Publikation in Betracht $\mathrm{zog}^{3}$.

Die Religion ist in Kants System der Philosophie nicht enthalten. Sie bildet ein isoliertes, eigenständiges Thema. So ist auch die Entstehung der Schrift "Die Religion innerhalb der Grenzen der bloßen Vernunft" durch ein anderes Interesse motiviert, nämlich durch die Frage der Abgrenzung der Philosophie und ihres Gegenstandes von der Theologie, welches schließlich das zentrale Thema im Fakultätenstreit zwischen der Theologie und der Philosophie ist. Zugleich bildet die Fakultätenschrift den Argumentationshintergrund für Kants Verteidigung und Zurückweisung der gegen ihn von der preußischen Staatszensur erhobenen Vorwürfe im Zusammenhang mit seiner Religionsschrift. Diese Vorgänge hat der Autor selbst in ihrer Chronologie sowie in ihrem logischen Zusammenhang im ersten Teil des "Streits der Fakultäten“ dargelegt. Aber schon in der Vorrede zur ersten Auflage der Religionsschrift selbst hat er dezidiert zur Sache Stellung genommen.

2 Zum Religions- und Zensuredikt vgl. Kant, 13:320-322. Vgl. außerdem: Arnoldt, Emil, „Beiträge zu dem Material der Geschichte von Kants Leben und Schriftstellertätigkeit in bezug auf seine ,Religionslehre' und sein Konflikt mit der preussischen Regierung", in: Altpreußische Monatsschrift, 34.5/6 (1894), 35.1/2 (1895) (auch als Buch unter gleichem Titel, Königsberg 1898; außerdem in: Emil Arnoldt, Gesammelte Schriften, Vol. 4, Berlin 1909); Dilthey, Wilhelm, „Der Streit Kants mit der Zensur über das Recht freier Religionsforschung", in: Archiv für Geschichte der Philosophie 3 (1890), S. 418-450 (wiederabgedruckt in Gesammelte Schriften, Vol. 4, Stuttgart, 2. Ausgabe, 1959, S. 285-309); Fromm, Emil, „Immanuel Kant und die preußische Censur, nebst kleinen Beiträgen zur Lebensgeschichte Kants“, Hamburg und Leipzig 1894; Fromm, Emil, „Zur Vorgeschichte der Königlichen Kabinetsordre an Kant vom 1. Oktober 1794", Kant-Studien 3 (1899), 142-147.

3 Vgl. R. Brandt, Zum „Streit der Fakultäten“ (1987), 63. 
Kants Neubestimmung des auf rein praktischer Vernunft basierenden moralischen Religionsbegriffs ist das Kriterium jener Grenzbestimmung, das eben genau durch die Autonomie des moralischen Gesetzes die theologische Fremdherrschaft über die Philosophie beendet und zugleich die traditionelle Stellung der Religion innerhalb der Metaphysik verändert.

Betrachtet man die erste Vorrede zur Religionsschrift, so ergeben sich drei Gesichtspunkte, die m.E. von hauptsächlicher Bedeutung sind: 1) die Forderung des Zensurgehorsams - d.i. der politische Aspekt; 2) das Verhältnis der "philosophischen Religionslehre“ zur Theologie, welches indirekt ebenfalls ein politischer Aspekt ist; 3) die Bestimmung der Religion als Verlängerung der Moral.

Offensichtlich hält Kant eine Zensurkontrolle unter staatlicher Oberaufsicht für erforderlich, um die Achtung gegenüber der Person und der Sache aus Freiheit erst möglich zu machen ${ }^{4}$. Diese Kontrolle kann aber nicht einseitig auf bloßer Fremdkontrolle beruhen, sondern schließt formal die Freiwilligkeit oder Selbstkontrolle ein. Mir scheint, daß diese Forderung Kants nur nachvollziehbar ist unter Berücksichtigung des Doppelcharakters des moralischen Gesetzes als einerseits auf freier Kausalität der Vernunft beruhend, andererseits die Nötigung notwendig (als Imperativ) enthaltend.

Die Zensur ist ein moralisches Gehorsamsgebot gegenüber der (staatlichen) „Obrigkeit“. Der politische und kulturpolitische Gesichtspunkt kommt hier ins Spiel durch das Interesse der Staatsführung an zuverlässigen und loyalen Priestern und Schullehrern für die Verwaltung, deren Ausbildungsort traditionell die Theologenfakultät ist. Die Beobachtung aller Pflichten „kann“ als Aufgabe der Religion betrachtet werden. Deshalb soll mit der Schrift, die die Religion zum Gegenstand hat, selbst ein Beispiel dieser Art von Zensur gegeben werden, d.h. Kant befürwortet im Prinzip die Zensur seiner eigenen Schrift; er ist nicht schlechthin gegen Zensur eingestellt, sondern hält sie im Gegenteil für notwendig. Aber er weist zugleich auf notwendige Bedingungen hin, die bei der Ausübung des Zensurgebotes strikt zu beachten sind. Die wesentliche Bedingung ist, daß die mit dem gebotenen Gehorsam verbundene, aus der moralischen Verpflichtung folgende "Achtsamkeit" nicht als eine solche gegen ein isoliertes Gesetz „im Staat“ mißzuverstehen ist, sondern „,nur durch vereinigte Achtung für alle vereinigt bewiesen werden“ kann (6:8). Der Zweck des Zensurgebots als Gehorsamsgebots ist also ein allgemeiner,

4 Stiening, G.: Die „Freiheit der gelehrten Feder" und der „Strich des Censors“. Immanuel Kant und die Universitätszensur. In: Brandt, R. / Euler, W. (Hrsg.), Studien zur Entwicklung preußischer Universitäten. Wiesbaden 1999 (Reihe, Wolfenbütteler Forschungen' Bd. 88), 163-202. Allgemein vgl. Losurdo, Domenico: Autocensura e compromesso nel pensiero politico di Kant, Napoli 2007. 
nämlich die moralische Achtung für das Gesetz überhaupt, dem alle als Untertanen gemeinsam verpflichtet sein sollen. Die Achtung ist zufolge der Kritik der praktischen Vernunft und der Konzeption der Tugendlehre in der Metaphysik der Sitten ein moralisches Gefühl, das "Triebfeder" moralischen Handelns ist.

Die Zensurausübung wird von Kant auf folgende Weise rekonstruiert: Der Zensor (oder ,Bücherrichter') erscheint in zweifacher Gestalt als Repräsentant der Theologie, die damit eine Doppelzensur ausübt: Der seelenheilende Priester wird in seiner Bücherzensur zugleich vom Wissenschaftskurator seiner Fakultät kontrolliert. Dieser letzte, der Gelehrte der theologischen Fakultät, ist als ,Oberzensor' berechtigt und beauftragt, als Glied der Universität „,alle Wissenschaften zur Cultur und zur Verwahrung gegen Beeinträchtigung" zu überwachen. Da der Theologe aber in beiden Funktionen ,Bibel-Theologe' ist, so wird die Selbstkontrolle der Theologie zum Problem, und es kann geschehen, daß er "um den Stolz der Wissenschaften zu demüthigen", in alle Wissenschaftsgebiete als Vormund eindringt. Es geht Kant darum, diese Grenzüberschreitung durch eine „Obercensur" zu verhüten (6:8).

Von der "biblischen Theologie" unterscheidet Kant eine "philosophische Theologie“, die in die Philosophie gehört. Das Verhältnis beider Fakultäten zueinander (als der obersten zur unteren), bestimmt sich in Hinsicht auf das Zensurrecht in doppelter Weise: einerseits hat die philosophische das Recht, alle Gegenstände der Theologie mitzubenutzen (selbst aus der Bibel zu zitieren), unter der Bedingung, daß sie „innerhalb der Grenzen der bloßen Vernunft bleibt" (6:9). Insofern hat die Philosophie "volle Freiheit" sich auf fremden Wissenschaftsgebieten umzusehen und auszubreiten. Sie ist aber hinsichtlich des Verwendungszwecks klar beschränkt: sie darf die aus der Theologie geschöpften Materialien „nur für sich“ benutzen, d.h. es ist ihr nicht gestattet, die philosophische Lehrmeinung in die Theologie "hineinzutragen" und das Privileg der geistlichen Lehre der Theologie anzutasten. Das Zensurrecht der theologischen Fakultät hat die Funktion, eine solche Grenzüberschreitung zu verhüten (und deshalb soll es bleiben), und zwar hat sie hier nur in diesem Falle das ausschließliche Recht der „erste[n] Zensur“ , d.h. der Oberzensur, und dieses hat die philosophische zu respektieren und sich ihr damit zu unterwerfen. Denn umgekehrt geht das Recht der Theologie nicht so weit, sich nun ihrerseits Eingriffe in die philosophische Verwendung theologischer Begriffe zu erlauben. Ihre Zensurfunktion umfaßt in Hinsicht auf die Philosophie nur die Aufgabe zu verhüten, daß jene in ihr Gebiet fremde Begriffe „hineinträgt" (6:10). Sie dient also der Selbstverteidigung ihrer Unabhängigkeit.

Indem die Philosophie mit der Moraltheologie sozusagen eine eigene, genuine, von der praktischen Vernunft getragene Religionslehre hat, ist sie gegen die Bevormundung durch die Theologie geschützt. 
Eine Abgrenzung der Philosophie von den übrigen Wissenschaften und ihren spezifischen Gegenständen, ist Kants Anliegen auch in anderen Fragestellungen, u.a. im Verhältnis zur Medizin, zu den Rechtswissenschaften, zur Mathematik. Zu Recht kann er deshalb auch in seinem Verteidigungsschreiben gegen die preußische Zensurbehörde darauf verweisen, dass ihn der Vorwurf der Einmischung der Philosophie in Fragen der Theologie am wenigsten treffen könne, da gerade er den Fehler der Grenzüberschreitung ,,jederzeit gerügt und dawider gewarnt habe" (7:7).

Der Eingriff der Philosophie in die Theologie geht aber immerhin so weit, daß Kant ihr prophezeien kann, daß sie ohne oder sogar gegen die Vernunft auf Dauer nicht bestehen werde. Er sieht sich ermutigt, den Absolventen der Theologie anzuraten, an ihr Studium der biblischen Theologie „noch eine besondere Vorlesung über die reine philosophische Religionslehre" als notwendige Vervollständigung ihrer Ausbildung anzuschließen. „Leitfaden“ dafür könnte ein Buch wie die vorliegende Religionsschrift des Autors sein (6:10.20-27). Eine solche Koalition zwischen Bibeltheologie und philosophischer Theologie (moralischer Religion) wird erst durch die scharfe Trennung und Vervollständigung ihrer jeweiligen Wissenschaften möglich. Damit ist zugleich das Feld für das Konkurrenzverhältnis im Streit der Fakultäten eröffnet.

In der Fakultätenschrift nimmt die Betrachtung des Streits zwischen der theologischen und der philosophischen Fakultät relativ breiten Raum ein. Die „Materie“ ihres Streits läßt sich am Gegensatz von „Kirchenglauben", der auf Statuten und äußeren Gesetzen beruht und vom biblischen Theologen vertreten wird, und dem "Religionsglauben" des „Vernunftgelehrte[n]“, der auf inneren Vernunftgesetzen beruht, festmachen. In dieser Hinsicht ist die Theologie als „Inbegriff gewisser Lehren als göttlicher Offenbarungen“ von der Religion als Inbegriff "aller unserer Pflichten überhaupt als göttlicher Gebote" unterschieden (7:36). Der Unterschied zwischen Religion und Moral ist hierbei nur ein formaler, behauptet Kant, nämlich daß Religion „eine Gesetzgebung der Vernunft [ist], um der Moral durch die aus dieser selbst erzeugte Idee von Gott auf den menschlichen Willen zu Erfüllung aller seiner Pflichten Einfluß zu geben" (7:36).

Kants Vorschlag zur religionsphilosophischen Erweiterung des Theologiestudiums trägt übrigens dem historischen Umstand Rechnung, daß in den staatlichen Verordnungen zur akademischen Ausbildung seit 1735 bereits festgelegt war, welche Auflagen und Beschränkungen die Philosophie im Verlauf ihrer Beschäftigung mit Fragen der Theologie $\mathrm{zu}$ beachten hatte ${ }^{5}$. Diese Bestimmungen werden in Kants Fakultäten-

5 S. dazu die „Erneuerte und erweiterte Verordnung, wie es in denen lateinischen Schulen, bey der Universität, mit denen Beneficiis und Stipendiis, mit der Wahl 
schrift aber nicht einfach wiederholt oder gar persifliert, sondern im Spannungsverhältnis zwischen Staatsräson und Autonomie der Wissenschaften und ihrer Institutionen neu reflektiert. Der Streit zwischen der philosophischen und der theologischen Fakultät ist der inneruniversitäre Schausplatz jener Auseinandersetzung zwischen Kirchenglaube und Religions- oder Vernunftglaube.

\section{Begriff und Organisation der Universität}

Die Universität ist ihrem Begriff nach bei Kant „eine Art von gelehrtem gemeinem Wesen" (7:17; vgl. 7:19.21) und damit eine Rechtsgemeinschaft. Denn das gemeine Wesen in Kants Staatslehre ist definiert als die Form eines Ganzen von Mitgliedern, die durch das gemeinsame Interesse Aller vereinigt sind, in einem rechtlichen Zustand leben zu wollen (RL, § 43). Das Hauptmerkmal der Universität ist somit ihre Autonomie. Diese besteht darin, dass die Gelehrten (Professoren) ihre Angelegenheiten selbst beurteilen. Die Universität ist so organisiert, daß sie mittels der „Facultäten“ die Funktionen zu erfüllen vermag, Schulabsolventen als „Lehrlinge“ aufzunehmen sowie Lehrer zu examinieren und als „Doctoren" zu graduieren.

Neben den normalen Gelehrten beherbergt sie auch eine Klasse der Nichtgelehrten, der sog. „Litteraten" oder "Geschäftsleute", die ein „bürgerliches $\mathrm{Amt}^{\prime \prime}{ }^{6}$ bekleiden (7:18), wie z.B. die Geistlichen. Sie sind „Werkzeuge der Regierung". Deshalb sind sie nicht frei in ihrem Urteil, sondern stehen, indem sie von den Wissenschaften öffentlichen Gebrauch machen, „unter der Censur der Fakultäten“. Indem sie sich unmittelbar ans Volk wenden, haben sie zwar "gesetzlichen Einfluß" auf das "Publicum “, aber sie haben in ihrem Fach nicht die "gesetzgebende", sondern nur die "ausübende Gewalt" in den Händen. Deshalb werden sie von der Regierung beaufsichtigt und befehligt, die darüber wacht, dass sie die richterliche Gewalt, die von den Fakultäten wahrgenommen wird, respektieren.

der Diaconorum an denen Kirchen, und der Rectorum und Präceptorum an denen Schulen, imgleichen mit anderen zum Kirchen- und Schulwesen gehörigen Dingen, in dem Königreich Preußen zu halten." In: Daniel Heinrich Arnoldts ausführliche und mit Urkunden versehene Historie der Königsbergischen Universität. Erster Theil, 1746, Beylage 54, S. 314 ff. Näheres in: Euler, W., Kants Amtsgeschäfte an der Universität Königsberg I, die vier Fakultäten, 1. Kapitel, Einleitung (Manuskriptfassung).

6 Vgl. Stiening, G.: Kants Begriff des öffentlichen Amtes, oder "Staatsverwaltung“ zwischen Aufklärung und Rechtsstaatlichkeit. In: Jahrbuch für europäische Verwaltungsgeschichte 19: Räte und Beamte in der Frühen Neuzeit. Lehren und Schriften. Baden-Baden 2007, 141-169. 
Man kann diese Gliederung auf Kants Gewaltenteilungslehre in der RL der MS beziehen7. Die Rolle der Geistlichen besteht dann der Analogie nach darin, einen Teil der „vollziehenden“ Gewalt zu bilden, dem die Staatsverwaltung obliegt, die (wie z.B. die Minister) die Befehle des Regenten, der die Gesetze ausführt, entgegennimmt und praktiziert (RL, $\S 49)$.

Die Einteilung der Fakultäten ergibt zwei Klassen: drei obere Fakultäten, eine untere (7:18-20). Das Unterscheidungsmerkmal, das seinen Ursprung in einer politischen Entscheidung (der Regierung) hatte, ist ein unterschiedliches Interesse: die Lehrvorträge der oberen liegen im Interesse der Regierung, die der unteren liegen allein im Interesse der Wissenschaft. Da das größte Interesse der Regierung darin besteht, sich den stärksten und dauerhaftesten Einfluß auf das Volk zu verschaffen, so besorgt sie sich am meisten um die Gegenstände der oberen Fakultäten. „Daher behält sie sich das Recht vor, die Lehren der oberen selbst zu sancioniren; die der untern überläßt sie der eigenen Vernunft des gelehrten Volks" (7:19.5-7). Aufgrund der Gewaltenteilung kann die Regierung aber nicht selbst für die Pflege der Wissenschaften sowenig wie für die Verbesserung der Lehre eintreten. Sie kann nicht die Rolle eines Supergelehrten spielen wollen.

Kant behauptet nun aber, es müsse ausser den betrachteten oberen Fakultäten noch eine solche geben, die als „zum gelehrten gemeinen Wesen" der Universität notwendig gehörend, in ihrer Lehre frei, d.h. unabhängig von der Befehlsgewalt der Regierung ist. Ihr kommt selbst keine Befehlsgewalt zu, aber doch die Freiheit, alle Befehle zu beurteilen, sofern sie „mit dem wissenschaftlichen Interesse, d.i. mit dem der Wahrheit" in Beziehung stehen. Die Vernunft ist berechtigt, in dieser Angelegenheit „öffentlich zu sprechen“. Diese Funktion ist notwendig zur Wahrheitsenthüllung, von der die Regierung profitiert. Die Ursache dafür, dass die philosophische Fakultät dennoch ,die untere' genannt wird, sieht Kant in der Natur des Menschen gegeben, ,daß nämlich der, welcher befehlen kann, ob er gleich ein demüthiger Diener eines andern ist, sich doch vornehmer dünkt als ein anderer, der zwar frei ist, aber niemanden zu befehlen hat" (07:20.9-11). Die Geschicklichkeit zum Befehlen bei gleichzeitiger Unterwerfung unter den Oberbefehl der Regierung steht den oberen Fakultäten und ihren Repräsentanten gut an und macht ihren Eigendünkel gegenüber der philosophischen Fakultät aus, der keine Befehlsgewalt zukommt. Von der Regierung ist aus der Sicht

7 Zu Kants Gewaltenteilungslehre vgl. M. Wolff: Trias politica. Erläuterungen zu Kants Verfassungstheorie in seinen Metaphysischen Anfangsgründen der Rechtslehre. In: W. Euler / B. Tuschling (Hrsg.), Kants „Metaphysik der Sitten“ in der Diskussion. Ein Arbeitsgespräch an der Herzog August Bibiliothek Wolfenbüttel 2009. Berlin 2013, $57-70$. 
der unteren Fakultät nur zu erwarten, dass sie die Rahmenbedingungen setzt für einen so weit wie möglich ungehinderten Fortgang der Wissenschaften (s. die zweite Fußnote, 7:19-20).

\section{Die Institutionentheorie der Universität}

Kants Auffassung über den Gliederbau der Universität und speziell über die Frage des Verhältnisses zwischen der theologischen und der philosophischen Fakultät gehört in den Rahmen seiner Institutionentheorie, die in kompakter Form in den Anfangspassagen des ersten Abschnittes der Fakultätenschrift dargestellt wird.

Institutionen (,künstliche[n] Einrichtungen“) liegt - nach dem Muster einer "Regierung" in der Staatstheorie - eine "Vernunftidee“ zu Grunde, deren Realität am vorhandenen Zustand der "Gelehrsamkeit“ als dem entsprechenden Gegenstand der Erfahrung "praktisch“ bewiesen werden soll. Die Gliederung einer Institution im allgemeinen wie auch der Universität im besonderen unterliegt somit keinem Zufall, sondern erfolgt „,nach irgend einem in der Vernunft, wenn gleich nur dunkel, liegenden Princip und darauf gegründetem Plan [...] der eine gewisse Art der Eintheilung nothwendig macht" (7:21.10-12).

Aus der Notwendigkeit der Vernunftidee der Universität schließt Kant auf die ursprüngliche Planung der Organisation der Lehranstalt seitens der Regierung, die nicht aus deren "Weisheit und Gelehrsamkeit" erfolgt sei, sondern auf dem "gefühlten Bedürfnis“ beruhte, „vermittelst gewisser Lehren aufs Volk zu wirken“. Dadurch sei sie auf ein „Princip der Eintheilung“ gebracht worden, „das mit dem jetzt [in der vorliegenden Abhandlung] angenommenen glücklich zusammentrifft" (7:21.15-21).

Es gibt eine Ordnung der Fakultäten nach den Motiven („Triebfedern") der Regierung, durch die sie ihren Zweck der Einflußnahme auf das Volk zu dessen „Wohl“ zu erreichen sucht, nämlich 1) das ewige, 2) das bürgerliche und 3) das leibliche Wohl. Für das ewige Wohl zu sorgen bedeutet dann, daß die Regierung durch die Kontrolle der öffentlichen Lehre danach trachtet, bei den Untertanen „das Innere der Gedanken" zu erkunden und "die verschlossensten Willensmeinungen“ zu „lenken“ (7:21.24-22.2). Die angegebene Reihenfolge der oberen Fakultäten entspricht zugleich der Vernunftordnung: theologische, juristische, medizinische Fakultät.

Wie die Universität als Ganzes so verfügen auch die Fakultäten über eigene Gesetzbücher oder "Statuten“. Aber nur die Universität als solche hat eine Verfassung. 


\section{Die Verfassung}

Die drei oberen Fakultäten erhalten einen Lehrauftrag von der Regierung in Verbindung mit vorgeschriebenen Lehrbüchern. Damit wird die Lehre normiert, d.h. sie wird für jeden aus dem Volk zugänglich und verläßlich (7:22.17-21). Schriftlich fixiert ist die "Norm" in "Statuten". Statute sind Lehrstücke (Anweisungen), die auf der „Willkür eines Obern“ beruhen und insofern von der Regierung sanktioniert sind und Gehorsam verlangen. Das Gleiche gilt auch vom "Gesetzbuch" der philosophischen Fakultät, obwohl deren öffentliche Lehre auch aus der Vernunft abgeleitet werden könnte. Soweit handelt es sich um die Gesetzbücher als „Kanon"8. Aber Kant erwähnt noch einen anderen Typ von Gesetzbüchern, die vermeintlich als Exposé im Geiste „des“ Gesetzbuches „zum faßlichern Begriff und sicherern Gebrauch des gemeinen Wesens (der Gelehrten und Ungelehrten)“ (7:22.31-32) von den Fakultäten selbst verfaßt sind. Solche Bücher besitzen nach Kant "gar keine Autorität". Denn sie haben anstelle einer Norm nur den pragmatischen Zweck, als Werbemittel der Fakultäten für die Aufnahme von Studenten zu dienen. Im Hinblick auf das Materiale der Gesetzgebung sind sie substanzlos. Sie betreffen höchstens die Didaktik, d.i. eine zeitlich beschränkt geltende Lehrmethode ("das Formale des Vortrags“). Folglich entnimmt der „biblische Theolog" als Repräsentant der obersten Fakultät seine Lehre nicht aus der Vernunft sondern aus der Bibel. Sollte er sich dazu verleiten lassen, seiner Lehre etwas beizumischen, was aus der Vernunft entlehnt ist, so verletzt er damit namens seiner Fakultät „die Autorität der durch sie [die Fakultät] gebietenden [geistlichen] Regierung und kommt ins Gehege mit der philosophischen“" (7:23.14-16). Deshalb ist den oberen Fakultäten insgesamt anzuraten, zur philosophischen auf Distanz zu gehen, um das „Ansehen ihrer Statute“ vor den "freien Vernünfteleien“ der unteren Fakultät zu schützen.

\section{Verhältnis der theologischen zur philosophischen Fakultät: "Geschäftsleute"}

Die Besonderheit der theologischen Fakultät wird von Kant anhand des Aufgabenfeldes dessen, den er den „biblischen Theologen“ nennt, ermittelt (ab 7:23.24). Der Theologe setzt in der Auslegung der Heiligen Schrift die Existenz Gottes durch das Medium der Bibel selbst voraus. Er

8 „Kanon“ nennt Kant den „Inbegriff der Grundsätze a priori des richtigen Gebrauchs gewisser Erkenntnisvermögen überhaupt" (KrV, B 824) 
kann und darf sich nicht in rationale Gottesbeweise versteigen, um den Ursprung des Göttlichen ergründen zu wollen, und sich schon gar nicht eine Art privater Moralphilosophie zurechtlegen und sie zum Gegenstand seines „öffentlichen Vortrage[s] ans Volk“ (7:23.35) machen, weil das „eine Sache der Gelehrsamkkeit“ ist, wovon das Volk nichts versteht und deshalb „nur in vorwitzige Grübeleien und Zweifel verwickelt werden würde“ (7:24.1-3). Er hat nicht eine Tugendlehre zugrunde zu legen, sondern kann sich nur auf ein unerklärliches „Gefühl der Göttlichkeit" und der "Gnade“ berufen (7:23.33, 34.11-15). Sobald der biblische Theologe seine Sätze mit Vernunft vermischt, „überspringt er [...] die Mauer des allein seligmachenden Kirchenglaubens und verläuft sich in das offene, freie Feld der eigenen Beurtheilung und Philosophie, wo er, der geistlichen Regierung entlaufen, allen Gefahren der Anarchie ausgesetzt ist" (7:24.18-25). Deshalb soll er ,reiner' Theologe bleiben, d.h. sich nicht infizieren an einem vermeintlichen „Freiheitsgeist der Vernunft und Philosophie" (7:24.27).

Der "Begriff" von der "unteren“, der philosophischen Fakultät, den Kant vorstellt, ist in sich zwiespältig (ab 7:27.13). Auf der einen Seite besteht ihr Kennzeichen gerade darin, sich nur mit Lehren zu beschäftigen, sofern sie „nicht auf den Befehl eines Oberen zur Richtschnur angenommen werden" (7:27.13-15). Auf der anderen Seite ergibt sich selbstverständlich auch für den Philosophielehrer eine Gehorsampflicht gegenüber der Obrigkeit daraus, dass er Mitglied einer staatlich sanktionierten Institution ist. Er kann sich daher, etwa bei der Wahl seines Gegenstandes (vorgeschriebener Lehrbücher) diesem Gehorsam nicht entziehen. Unvereinbar mit dem Begriff und der Funktion der Philosophie als Fakultät wäre es hingegen, wenn auch die "Wahrheit" des öffentlichen Lehrvortrages durch den Befehl besiegelt werden sollte. Dem oben angedeuteten Zwiespalt scheint Kant jedoch dadurch ausweichen zu wollen, dass er verlangt, daß ein seine Lehre betreffender, an den Philosophen ergangener Befehl auf dessen Einsicht beruhen muss. Dieses "freie[] Urtheil" soll es erlauben, die Autorität der Vernunft über die des Staates zu stellen, so dass sozusagen, die Berufsehre des Philosophen gerettet ist. Kants Fazit lautet also mit Bezug auf die untere Fakultät:

"Also wird die philosophische Facultät darum, weil sie für die Wahrheit der Lehren, die sie aufnehmen oder auch nur einräumen soll, stehen muß, in so fern als frei und nur unter der Gesetzgebung der Vernunft, nicht der der Regierung stehend gedacht werden müssen“ (7:27.32-35).

Die notwendige Einrichtung einer philosophischen Fakultät an der Universität ergibt sich aus ihrer Funktion, die oberen Fakultäten kontrollieren zu sollen. Sie überprüft den Inhalt ihrer Lehren in bezug auf das Kritierium der Wahrheit, welche die „erste Bedingung der Gelehrsamkeit überhaupt" ist (7:28.4-5). Der zweite Gesichtspunkt ist der des Nutzens. Durch die Wahrheitsprüfung verschafft die philosophische Fakultät den 
drei oberen den Nutzen, dass sie so dem Zweck der Regierung besser zugänglich und dienlich sein können. Und das ergibt sich aus Kants Perspektive aus der "Anspruchslosigkeit" der Philosophie, „blos frei zu sein, aber auch frei zu lassen, blos die Wahrheit zum Vortheil jeder Wissenschaft auszumitteln und sie zum beliebigen Gebrauch der oberen Facultäten hinzustellen" (7:28.12-14). Denn dadurch empfiehlt sie sich der Regierung gegenüber „als unverdächtig, ja als unentbehrlich" (7:28.14-15).

Weil nun die philosophische Fakultät in sich noch einmal in zwei Departements unterteilt ist, nämlich in eine historische und eine VernunftAbteilung der Erkenntnis, wobei die letztere das Gebiet der Philosophie im engeren Sinne umfaßt, so erstreckt sie sich - wenigstens historisch „auf alle Teile des menschlichen Wissens" (7:28.22-23). Sie kann sich das aber nicht auch zum Inhalt der eigenen Lehre machen, sondern ist nur berechtigt, die Gegenstände der Lehre der anderen Fakultäten ihrer Prüfung und Kritik zu unterwerfen, um den Nutzen der Wissenschaften zu befördern.

Die Aufgabe der Wahrheitsstiftung verleiht der philosophischen Fakultät Immunität gegenüber der Regierung. Es kann kein Lehrverbot über sie verhängt werden (denn damit würde die Regierung ihrem eigenen Zweck zuwiderhandeln), und die oberen Fakultäten müssen ihre Kritik respektieren (7:28.29-35).

Es sind in den oberen Fakultäten allein die Geschäftsleute, denen nach Kant verwehrt werden muß, sich in ihrem Lehrvortrag als Philosophen zu betätigen; denn sie handeln als „Beamte“ (hier: als Prediger) kraft ihres Amtes im Auftrag der Regierung. Dagegen dürfen die Fakultäten, von denen die Beamten ihre Ausbildung und ihr Wissen erwerben, nach dem Vorbild der Philosophen Kritik an den offiziellen Lehren üben. Kants Argumentation für diese Option ist die folgende: wenn die Staatsbeamten ihre Einwände "gegen die geistliche oder weltliche Gesetzgebung" dem Volk nahebringen, dann wiegeln sie dies dadurch gegen die Regierung auf. Wenn der Konflikt aber auf der Gelehrtenebene der Fakultäten und unter diesen selbst ausgetragen wird, dann ist das ungefährlich. Denn das Volk interessiere sich in der Regel nicht für Gelehrtenstreitigkeiten (,weil es sich selbst bescheidet, daß Vernünfteln nicht seine Sache sei, und sich daher verbunden fühlt, sich nur an dem $\mathrm{zu}$ halten, was ihm durch die dazu bestellte Beamte der Regierung verkündigt wird" (7:29.11-13)). In der weiteren Konsequenz führt die Freiheit der unteren Fakultät dazu, „daß die obern Facultäten“, da sie durch den Wahrheitsanspruch der unteren besser belehrt sind, "die Beamte immer mehr in das Gleis der Wahrheit bringen, welche dann ihrerseits, auch über ihre Pflicht besser aufgeklärt, in der Abänderung des Vortrags keinen Anstoß finden werden“ (7:29.13-18). D.h. durch den Ein- 
fluß der Philosophie werden auch Staatsbeamte offener für Kritik und flexibel in der Handhabung ihrer Pflichten, „welches ohne polemische und nur Unruhe erregende Angriffe auf bisher bestandene Lehrweisen mit völliger Beibehaltung des Materialen derselben gar wohl geschehen kann" (7:29.19-21). Mittelbar profitiert also die Regierung immer durch den Wahrheitsanspruch der philosophischen Fakultät, indem diese durch die Kontrolle der oberen Fakultäten den Beamtenapparat zu einem höchst effizienten Instrument der Regierung macht. - Soweit Kants Vorstellung von einer Aufklärung, von unten'. Was die Fakultäten aber auf diese Weise unter sich austragen sollen, ist eine politische Antinomie, ein (nicht auflösbarer) Interessenskonflikt, der in der Autorität der Staatsführung selbst seine Wurzeln hat und der in der Gegensätzlichkeit von Herrschafts- und Freiheitsanspruch besteht.

\section{Der gesetzwidrige Streit}

Beim Streit der Fakultäten geht es der Sache nach „um den Einfluß aufs Volk", d.h. darum, dem Volk durch das am besten geeignete Mittel glaubhaft zu machen, daß man sein Wohl besorgen will. Dieser Gelehrtenstreit (als ein „öffentlicher Streit der Meinungen“) ist gesetzwidrig entweder der Materie nach, insofern sein Gegenstand gar nicht für eine öffentliche Debatte zulässig ist; oder der Form nach, wenn die Art der Auseinandersetzung nicht objektiv und rational ist, sondern aus Urteilen mit subjektiven Beweggründen besteht ${ }^{9}$. Solche Urteile werden aus Neigung gefällt, um den Gegner "durch List (wozu auch Bestechung gehört) oder Gewalt (Drohung) zur Einwilligung zu bringen“ (7:29.25-33).

Es handelt sich hierbei nicht um das Volk als Gesetzgeber oder Souverän, sondern um die Menge der Untertanen. Denn Kant sagt über dasselbe, daß es sein Wohl („Heil“) nicht zuerst in die Freiheit sondern in die drei natürlichen Zwecke setze, 1) der Seligkeit nach dem Tod, 2) der Sicherung des individuellen Besitzes im sozialen Umfeld durch öffentliche Gesetze, 3) des physischen Lebensgenusses (der Gesundheit und eines langen Lebens) (7:30.5-9). Diese Trias korrespondiert der weiter oben festgestellten Trias der Motive der Regierung zu ihrem eigenen Zweck (vgl. 7:21.22-22.5).

Die philosophische Fakultät aber stellt sich quer zu dieser Entsprechung zwischen den Erwartungen des Volkes und den Motiven der Regierung. Sie beantwortet die Wünsche und Begehrungen des Volkes,

9 Vgl. R. Brandt: Universität zwischen Selbst- und Fremdbestimmung. Kants ,Streit der Fakultäten'. Mit einem Anhang zu Heideggers ,Rektoratsrede'. Berlin 2003 (Deutsche Zeitschrift für Philosophie. Sonderband 5), 62-64. 
indem sie mit Vorschriften, die sie aus der Vernunft (und nicht aus der Neigung) schöpft, darauf reagiert. Da ihre Vorschriften dem Prinzip der Freiheit folgen, bestehen die Anworten nur aus dem, „was der Mensch selbst hinzuthun kann und soll"; das ist: "rechtschaffen zu leben, keinem Unrecht zu thun, sich mäßig im Genusse und duldend in Krankheiten und dabei vornehmlich auf die Selbsthülfe der Natur rechnend zu verhalten" (7:30.13-15). Um diesen Forderungen nachzukommen, bedarf es keiner besonderen Gelehrsamkeit, sondern nur der Beherrschung der Neigungen durch die eigene Vernunft. Diese zu gebrauchen, lehrt die philosophische Fakultät.

\section{Verhältnis zwischen Volk und Gelehrtentum: Wundermänner}

Das Volk wird von Kant hier so umschrieben, daß es um des Genusses seiner eigenen Neigungen willen von den oberen Fakultäten verlangt, Patentlösungen für das eigene Seelenheil trotz selbstverschuldeter Versäumnisse bereitzustellen:

„Es ist aber hier, als ob das Volk zu dem Gelehrten wie zum Wahrsager und Zauberer ginge, der mit übernatürlichen Dingen Bescheid weiß" (7:30.32-34).

Aufgrund dieser Erwartungshaltung ist es auf der anderen Seite nicht verwunderlich, daß sich das Volk von dem Versprechen von jemand, der sich als ein solcher "Wundermann" ausgibt, d.h. als jemand, der große Vergünstigungen und Glückstaten verspricht, ohne sein Urteil rational überprüft zu haben, angezogen fühlt und das ernsthafte Urteil der philosophischen Fakultät verwirft.

Solche Wundermänner rekrutieren sich zwangsläufig aus den „Geschäftsleuten" der oberen Fakultäten, sofern nicht zugelassen wird, dass sich ihnen die philosophische Fakultät entschlossen in den Weg stellt, um die Faszination ihrer Wirkung auf das Volk beim Volk als Täuschung (Betrug) zu entlarven. Dies scheint umso mehr in die Kompetenz der philosophischen Fakultät zu fallen, als die Regierung, anstatt auf der Grundlage der "Idee der Sittlichkeit“ den Enthusiasmus des Volkes zu bremsen, vielmehr „aus Furcht vor Kraftlosigkeit dieser Ideen“ auf den Einfall kommt, "für sie in Bildern und kindischem Apparat Hilfe zu suchen". Die Religion wird so indirekt ein Medium des Staates, das Volk gefügig, in seinem Sinne manipulierbar zu halten:

„Daher haben auch Regierungen gerne erlaubt, die Religion mit dem letzteren Zubehör reichlich versorgen zu lassen und so dem Untertan die Mühe, zugleich aber auch das Vermögen zu benehmen gesucht, seine Seelenkräfte über die Schranken auszudehnen, die man ihm willkürlich 
setzen, und wodurch man ihn [den Untertan], als bloß passiv, leichter behandeln kann" (KU, B 125).

Der Streit zwischen den oberen und der unteren Fakultät verwandelt sich dadurch in einen gesetzwidrigen, daß die oberen - entgegen ihrer eigentlichen Bestimmung - bewußt Gesetze mißachten und Grundsätze der Demagogie wählen, um sie durch ihre "große Kunst und Geschicklichkeit“" zur Weltverbesserung anzupreisen (7:31.11-17).

Der Konflikt im "gesetzwidrigen Streit" zwischen den Parteien der oberen und der unteren Fakultät, der Regierung und dem Volk hinsichtlich ihrer jeweiligen Erwartungen und Einflußmöglichkeiten stellt sich bei subtiler Betrachtung als ein komplexes Beziehungsfeld dar: Die Regierung kann nur indirekt auf das Volk wirken, nämlich mittels der oberen Fakultäten. Sie nutzt das Bedürfnis des Volkes nach Leitfiguren aus, die sie aus der oberen Fakultät rekrutiert und in Gestalt von deren "Geschäftsmännern" mobilisiert. Das sind „Praktiker", die „das Machwerk (savoir faire) verstehen“ (die das know-how besitzen: Geistliche, Justizbeamte, Ärzte). Durch diese Instrumentalisierung wird nun nach Kants Vorstellung die Regierung "selbst verleitet", "den Facultäten eine Theorie aufzudringen, die nicht aus der reinen Einsicht der Gelehrten derselben" stammt, sondern auf einem Kalkül über die Einflußmöglichkeiten auf das Volk mittels der "Geschäftsmänner" beruht. Dieses Kalkül stützt sich auf die Erkenntnis (Überzeugung), daß das Volk natürlicherweise denjenigen am meisten vertraut, die seine Ruhe und Bequemlichkeit nicht stören, also nicht etwa die Sache der Aufklärung vertreten und fordern, „sich selbst zu bemühen und sich seiner eigenen Vernunft zu bedienen“ (7:31.18-29) („das Volk will geleitet [...] sein“) (7:31.18-19).

Es scheint deshalb so, daß es im "Glauben“ (theologisch verstanden) am leichtesten möglich ist, „die Pflichten mit den Neigungen in Verträglichkeit“ zu bringen und so ohne Prüfung des Gegenstandes des Glaubens das Seelenheil zu erwerben (7:31.30-35).

Der charakterisierte gesetzwidrige Streit der Fakultäten ist endlos, und zwar weil „das Princip der Gesetzgebung“ für die oberen, das der Regierung zugeschrieben wird, in Wahrheit eine Gesetzlosigkeit ist, die von derselben Regierung autorisiert zu sein scheint (7:32.1-3). Eine solche Gesetzgebung ist aber deshalb gesetzlos, weil sich aus "Neigung" oder „Privatabsicht" allgemein kein Gesetz konstituieren kann. Deshalb können die oberen Fakultäten ein solches Gesetz auch gar nicht publizieren, und die Regierung kann es auch nicht sanktionieren, ohne gegen die Vernunft selbst zu verstoßen. Täte sie es dennoch, würde sie den Streit der oberen Fakultäten mit der philosophischen entfachen. Das aber soll verhindert werden, da in dessen Verlauf der philosophischen Fakultät und damit mittelbar auch dem Universitätsgebilde insgesamt die Zerstörung drohte. 


\section{Der gesetzmäßige Streit}

Unangesehen des Inhalts der Lehren, die die oberen Fakultäten im Auftrag der Regierung verbreiten, sind sie der Form nach nur als "Statute" aufzufassen, die der Willkür der Regierung entspringen ${ }^{10}$. Gleichwohl kann dieser die Wahrheit jener Lehren nicht gleichgültig sein. Sie müssen also der Vernunft "unterworfen bleiben“, die von der philosophischen Fakultät aus ihrem ureigenen Interesse heraus gepflegt wird. Eine solche Wahrheitspflege setzt unbedingt die Zulassung „völliger Freiheit einer öffentlichen Prüfung derselben [d.i. der Wahrheit]“ voraus. Da aber willkürlich geschaffene „Satzungen“ mit den notwendigen Vernunftlehren nicht ohne weiteres übereinstimmen, so wird deshalb der Streit der Fakultäten notwendig („unvermeidlich“) und zugleich "gesetzmäßig “11. Dabei ist die untere sogar verpflichtet, darauf zu achten, daß alles, was als "Grundsatz" gelten soll, ,wahr" ist (7:32.29-32).

Die philosophische Fakultät erhält hierdurch die verbindliche Aufgabe, erstens den historischen Quellen der jeweils sanktionierten Lehren (wie z.B. der biblischen Geschichte) mit dem Anspruch kritischer Überprüfung nachzuforschen; zweitens auch die rationalen Gründe der Gesetzgebung aus dem historischen Kontext herauszulösen und zu analysieren; und drittens die ästhetische Seite der Quelle der sanktionierten Lehren, nämlich ein damit verbundenes vermeintliches "Gefühl“ (wie ein „frommes Gefühl eines übernatürlichen Einflusses“) „,mit kalter Vernunft öffentlich zu prüfen“. Der Entschluß, „,dieses vermeinte Gefühl auf Begriffe zu bringen", steht der philosophischen Fakultät frei, ist ihre ungehinderte Aufgabe (7:33.11-15).

Es gibt vier „Formalgrundsätze“ bzw. Folgen, die die Austragung des gesetzmäßigen Streits der Fakultäten regulieren:

(1) Der Streit kann nicht durch eine "friedliche Übereinkunft" beigelegt werden, sondern muß durch den Richterspruch der Vernunft entschieden werden; denn nur dadurch wird das Urteil "rechtskräftig" (allgemein verbindlich).

(2) Der Streit hört nie auf (auch nicht durch den Richterspruch der Vernunft), weil es einerseits immer "statutarische Vorschriften" mit Bezug auf öffentliche Lehren geben muß. Denn - so Kant von der unbeschränkten Freiheit ,,alle seine Meinungen ins Publicum zu schreien“" geht eine Gefahr sowohl für die Regierung als auch für das Publikum aus. Andererseits aber sind auch die

10 Vgl. R. Brandt, Universität (2003), 59 f.

11 Der Streit der Fakultäten ist als „Auseinandersetzung zwischen Herrschaft und Wahrheit" - wie R. Brandt treffend bemerkt - "das Lebenselixier der Universität" (Brandt, ebd., 57). 
sanktionierten Satzungen der Regierung der Gefahr des Irrtums und der Zweckwidrigkeit ausgesetzt. Das betrifft also auch diejenigen Sanktionen, die an die oberen Fakultäten adressiert sind. Die philosophische Fakultät muß daraus ableiten, daß sie ununterbrochen wachen und ihre Schutzfunktion in Ansehung der Wahrheit aufrecht erhalten muß, um die nie zu befriedigende „Begierde zu herrschen“ der oberen Fakultäten abwehren zu können (7:33.25-37).

(3) In diesem Streit ist die Regierung neutral. Sie ist nicht direkt Streitgegner, sondern Zuschauer im Streit der Fakultäten untereinander. Insofern bleibt ihre Integrität gewahrt. Zwar schreibt sie den "Geschäftsleuten" der oberen Fakultäten bestimmte Sätze für deren öffentlichen Vortrag vor und stellt sie auch unter ihren Schutz, aber dieses Protektorat dient nur zu ihrem eigenen Vorteil, um nicht selbst über die Wahrheit in der Sache der Gelehrten entscheiden zu müssen. Umgekehrt sind die oberen Fakultäten der Regierung "für nichts verantwortlich“ außer für "die Instruction und Belehrung, die sie ihren Geschäftsleuten zum öffentlichen Vortrage geben" (7:34.11-14). Diese Verantwortlichkeit ergibt sich daraus, dass die an die Geschäftsleute in Auftrag gegebenen Vorträge an das Publikum gerichtet sind, das als „bürgerliches gemeines Wesen“ zu verstehen ist; während die Lehren der "Theoretiker" (der Wissenschaftler) aller Fakultäten von einem Publikum aufgenommen werden, das ein "gelehrte[s] gemeine[s] Wesen[]“ repräsentiert. Das Volk, das dieser Materie unkundig ist, gehört diesem Wesen nicht an, und die Regierung findet es nicht schicklich, sich mit wissenschaftlichem Lehrstoff zu befassen. Werden diese beiden Arten des gemeinen Wesens nicht auseinander gehalten - wird von den "Praktikern" das, was sie für Theorie halten, unter den Augen des „bürgerlichen gemeinen Wesen[s]“ verhandelt so wird der "gelehrte Streit" in einen "gesetzwidrigen" Streit verwandelt, „wo Lehren den Neigungen des Volks angemessen vorgetragen werden und der Same des Aufruhrs und der Factionen ausgestreut, die Regierung aber dadurch in Gefahr gebracht wird" (s. Fn. 7:34).

Wenn Kant in diesem Kontext das Bild eines Gelehrtenparlaments entwirft, so setzt er darin die "Classe der obern Facultäten“, die die Statuten der Regierung verteidigt, an die „rechte“ Position, „die Bank der philosophischen Facultät" hingegen als die "Oppositionspartei“ an die "linke Seite“ (7:35.1-5). Auf der Grundlage einer "freien Verfassung", bei der es um die Wahrheit geht, definiert sich die Oppositionsrolle dadurch, dass sich die Regierung nicht selbst hinreichend belehren kann, da das Gelehrtentum nicht ihre Sache ist. Sie muß sich also durch die 
strenge Prüfung und die Einwände der philosophischen Fakultät belehren lassen.

Die Geschäftsleute nun, sofern sie in den von der Regierung ihnen übergebenen Vorträgen Änderungen vornehmen wollen, können nicht unmittelbar von jener gemaßregelt werden, sondern nur mittels eines von ihrer Fakultät verlangten "Gutachten[s]" (7:35.7-15).

(4) Der Streit der Fakultäten widerspricht nicht der friedlichen Koexistenz des "gelehrten" und des „bürgerlichen gemeinen Wesens", soweit es deren Maximen betrifft. Denn durch die Befolgung derselben, arbeiten sich beide "Classen von Facultäten“ kontinuierlich zu größerer Vollkommenheit vor. Das Ziel dieser Entwicklung ist die „Entlassung von allen Einschränkungen der Freiheit des öffentlichen Urtheils durch die Willkür der Regierung" (7:35.19-21). In Kants Vision ist die Philosophie die Partei, die als Siegerin aus dem Streit hervorgeht. Sie wird der theologischen Fakultät nicht die Macht entreissen - insofern wird die Fakultätenhierarchie nicht einfach umgekehrt - aber sie kann die entscheidende Stelle in der Regierungsberatung einnehmen, und die Regierung würde von ihrer Freiheit profitieren, da daraus Einsichten resultieren, in der sie bessere Mittel zur Erreichung ihrer Zwecke findet als ,,in ihrer eigenen absoluten Autorität" (7:35.22-27).

\section{Bibliography}

Arnoldt, Emil, „Beiträge zu der Material der Geschichte von Kants Leben und Schriftstellertätigkeit in bezug auf seine ,Religionslehre' und sein Konflikt mit der preussischen Regierung", in: Altpreussische Monatsschrift, 34.5/6 (1894), 35.1/2 (1895) (auch als Buch unter gleichem Titel, Königsberg 1898; außerdem in: Emil Arnoldt, Gesammelte Schriften, Vol. 4, Berlin 1909).

Bödeker, Hans Erich, „Von der ,Magd der Theologie` zur ,Leitwissenschaft'. Vorüberlegungen zu einer Geschichte der Philosophie des 18. Jahrhunderts“. In: Das achtzehnte Jahrhundert, Bd. 14 (1990), H. 1, 19-57.

Borowski, Ludwig Ernst, „Darstellung des Lebens und Charakters Immanuel Kants“, Königsberg 1804, Beilage „IV. Kants Zensurleiden“, in Felix Gross (Hg.): Immanuel Kant. Sein Leben in Darstellungen von Zeitgenossen. Die Bibliographien von L. E. Borowski, R. B. Jachmann und E. A. Ch. Wasianski. Mit einer Einleitung von Rudolf Malter. Darmstadt 1993.

Brandt, Reinhard: Zum „Streit der Fakultäten“. In: R. Brandt / W. Stark (Hrsg.), Neue Autographen und Dokumente zu Kants Leben, Schriften und Vorlesungen. Hamburg 1987 (Kant-Forschungen Bd. 1), 31-78.

Brandt, Reinhard / Euler, Werner (Hg.), Studien zur Entwicklung preußischer Universitäten. Wiesbaden 1999 (Reihe ,Wolfenbütteler Forschungen' Bd. 88). 
Brandt, Reinhard: Universität zwischen Selbst- und Fremdbestimmung. Kants ,Streit der Fakultäten'. Mit einem Anhang zu Heideggers ,Rektoratsrede'. Berlin 2003.

Dilthey, Wilhelm, „Der Streit Kants mit der Zensur über das Recht freier Religionsforschung", in: Archiv für Geschichte der Philosophie 3 (1890), S. 418-450 (wiederabgedruckt in Gesammelte Schriften, Vol. 4, Stuttgart, 2. Ausgabe, 1959, S. 285-309).

Fromm, Emil, „Immanuel Kant und die preussische Censur, nebst kleinen Beiträgen zur Lebensgeschichte Kants“, Hamburg und Leipzig 1894.

Fromm, Emil, „Zur Vorgeschichte der Königlichen Kabinetsordre an Kant vom 1. Oktober 1794“, Kant-Studien 3 (1899), 142-147.

Gerhardt, Volker (Hrsg.): Kant im Streit der Fakultäten. Berlin 2005.

Losurdo, Domenico: Autocensura e compromesso nel pensiero politico di Kant. Napoli 2007.

Stiening, Gideon, Die „Freiheit der gelehrten Feder“ und der "Strich des Censors". Immanuel Kant und die Universitätszensur. In: Brandt, R. / Euler, W. (Hrsg.), Studien zur Entwicklung preußischer Universitäten (1999), 163-201.

Stiening, Gideon, Kants Begriff des öffentlichen Amtes, oder "Staatsverwaltung" zwischen Aufklärung und Rechtsstaatlichkeit. In: Jahrbuch für europäische Verwaltungsgeschichte 19: Räte und Beamte in der Frühen Neuzeit. Lehren und Schriften. Baden-Baden 2007, 141-169.

\section{Summary}

\section{The Contest of Theological and Philosophical Faculties in "The Contest of Faculties" by Immanuel Kant}

Kant's mature work on the conflict of the faculties includes a theory of the basic establishment of philosophy as an institution embedded in the hierarchical structure of the four faculties in university and touched by the historical conditions of Prussian cultural policy at the end of the $18^{\text {th }}$ century. It involves substantially two intentions: $1^{\text {st }}$ to redefine its ranking within the system of the faculties, changing the position from the lowest to the highest level; $2^{\text {nd }}$ to defend in the name of all faculties the autonomy and the rights of university as a whole unity in relation to the political demands of the Prussian state, because the political autonomy of the university was held to be the precondition for the guaranty of independence of science. In both cases philosophy enters into a struggle with the clerical authority of theology on the one hand and political administration on the other. A central topic of the conflict was the meaning and the role of religion within academic and social life. On the background of these two lines of arguments it will be our tasks in this article $1^{\text {st }}$ to clarify the relation between philosophy and theology concerning moral religion, $2^{\text {nd }}$ to examine the practical relation between the university under the leadership of philosophy and the state 
as far as the political power should be enlightened about the true principles of government by philosophical reason.

\section{Keywords}

idea of university, conflict of the faculties, philosophy, theology, religion, political power, state 